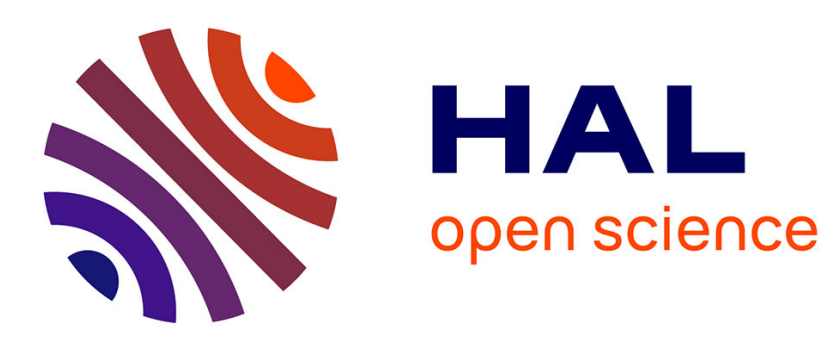

\title{
The gamma-core in Cournot oligopoly TU-games with capacity constraints
}

\author{
Aymeric Lardon
}

\section{To cite this version:}

Aymeric Lardon. The gamma-core in Cournot oligopoly TU-games with capacity constraints. 2009. halshs-00544042

\section{HAL Id: halshs-00544042 \\ https://shs.hal.science/halshs-00544042}

Submitted on 7 Dec 2010

HAL is a multi-disciplinary open access archive for the deposit and dissemination of scientific research documents, whether they are published or not. The documents may come from teaching and research institutions in France or abroad, or from public or private research centers.
L'archive ouverte pluridisciplinaire HAL, est destinée au dépôt et à la diffusion de documents scientifiques de niveau recherche, publiés ou non, émanant des établissements d'enseignement et de recherche français ou étrangers, des laboratoires publics ou privés. 


\title{
The $\gamma$-core in Cournot oligopoly TU-games with capacity constraints
}

\author{
LARDON Aymeric ${ }^{* \dagger}$
}

September 20, 2010

\begin{abstract}
In cooperative Cournot oligopoly games, it is known that the $\beta$-core is equal to the $\alpha$-core, and both are non-empty if every individual profit function is continuous and concave (Zhao 1999b). Following Chander and Tulkens (1997), we assume that firms react to a deviating coalition by choosing individual best reply strategies. We deal with the problem of the non-emptiness of the induced core, the $\gamma$-core, by two different approaches. The first establishes that the associated Cournot oligopoly TU(Transferable Utility)-games are balanced if the inverse demand function is differentiable and every individual profit function is continuous and concave on the set of strategy profiles, which is a step forward beyond Zhao's core existence result for this class of games. The second approach, restricted to the class of Cournot oligopoly TU-games with linear cost functions, provides a single-valued allocation rule in the $\gamma$-core called NP(Nash Pro rata)-value. This result generalizes Funaki and Yamato's core existence result (1999) from no capacity constraint to asymmetric capacity constraints. Moreover, we provide an axiomatic characterization of this solution by means of four properties: efficiency, null firm, monotonicity and non-cooperative fairness.
\end{abstract}

Keywords: Cournot oligopoly TU-games; $\gamma$-core; Balanced game; NP-value; Noncooperative fairness

\section{Introduction}

Usually, oligopoly situations ${ }^{1}$ are modeled by means of non-cooperative games. Every profit-maximizing firm pursues Nash strategies and the resulting outcome is not Pareto

${ }^{*}$ A. LARDON $(\bowtie)$ University of Saint-Etienne, GATE Lyon Saint-Etienne, CNRS, 6 rue Basse des Rives, 42023 Saint-Etienne, France, e-mail: aymeric.lardon@univ-st-etienne.fr, Tel: $(+33)(0) 4.77 .42 .19 .62$, Fax: $(+33)(0) 4.77 .42 .19 .50$

${ }^{\dagger}$ I wish to thank Theo Driessen, Anna Khmelnitskaya, Philippe Solal, Sylvain Béal, David Bartl, Pascal Billand and Christophe Bravard for providing numerous suggestions that substantially improved the exposition of the article. I have also benefited from comments of seminar participants at Twente University. Financial support by National Agency for Research (ANR) - research program "Models of Influence and Network Theory" ANR.09.BLANC-0321.03 - is gratefully acknowledged.

${ }^{1}$ In the remainder of this paper we use the term "oligopoly" to refer to the Cournot oligopoly. 
optimal. Yet, it is known that firms are better off by forming cartels and that Pareto efficiency is achieved when all the firms merge together. A problem faced by the members of a cartel is the stability of the agreement and non-cooperative game theory predicts that member firms have always an incentive to deviate from the agreed-upon output decision.

However, in some oligopoly situations firms don't always behave non-cooperatively and if sufficient communication is feasible it may be possible for firms to sign agreements. A question is then whether it is possible for firms to agree all together and coordinate their output decision to achieve Pareto efficiency. For that, we consider a fully cooperative approach for oligopoly situations without transferable technologies, i.e. cartel-wide production technology is determined by all member firms and not by the most efficient firm in the cartel. For studying properties of cooperative oligopoly games, we convert the associated normal form oligopoly game into an oligopoly TU-game in which firms can form cartels acting as a single player. Since the inverse demand function depends on the total output, the output decision of a cartel as well as its income depend on outsiders' behavior. Hence, the determination of the income that a cartel can obtain requires to specify how firms outside react. Aumann (1959) proposes two approaches: according to the first, every cartel computes the total profit which it can guarantee itself regardless of what outsiders do; the second approach consists in computing the minimal profit for which outsiders can prevent the players in the cartel from getting more. With or without transferable technologies, Zhao (1999a,b) shows that these two approaches lead to the same oligopoly TU-game by proving that the associated characteristic functions, called $\alpha$ - and $\beta$-characteristic functions respectively, are equal.

In oligopoly TU-games with transferable technologies, Zhao (1999a) provides a necessary and sufficient condition to establish the convexity property in case the inverse demand function and cost functions are linear. Although these games may fail to be convex in general, Norde et al. (2002) show they are nevertheless totally balanced. Concerning oligopoly TU-games without transferable technologies, Zhao (1999b) proves that the $\beta$-core is non-empty if every individual profit function is continuous and concave. ${ }^{2}$ Furthermore, Norde et al. (2002) show that these games are convex in case the inverse demand function and cost functions are linear, and Driessen and Meinhardt (2005) provide economically meaningful sufficient conditions to guarantee the convexity property in a more general case.

All these articles share the assumption that outsiders minimize the income of a deviating coalition ( $\alpha$ - and $\beta$-characteristic functions). However, this assumption can be questioned since outsiders probably cause substantial damages upon themselves by increasing their output at full capacity. A similar argument is developed by Rosenthal (1971). We propose to consider an alternative blocking rule suggested by Chander and Tulkens (1997) where external agents choose their action individually as a best reply to the coalitional action. This leads to consider the "partial agreement characteristic function" or, for short, $\gamma$-characteristic function. These authors analyse a game in coalitional

\footnotetext{
${ }^{2}$ Zhao shows that the $\beta$-core is non-empty for general TU-games in which every strategy set is compact and convex, every utility function is continuous and concave, and satisfying the strong separability condition that requires that the payoff function of a coalition and each of its members' utility functions have the same minimizers. Zhao proves that oligopoly TU-games satisfy this latter condition.
} 
form derived from an economy with environmental externalities and exhibit a strategy, the ratio equilibrium (Kaneko 1977), for which the resulting outcome belongs to the $\gamma$-core for linear utility functions. Helm (2001) generalizes their result by showing that the balancedness condition is satisfied when every individual utility function is differentiable and concave. Funaki and Yamato (1999) study an economy with a common pool resource that also describes an oligopoly situation and show that the associated cooperative game admits a non-empty $\gamma$-core in case firms have symmetric and linear cost functions and no capacity constraint.

In this paper we focus on oligopoly TU-games without transferable technologies. Our main objective is to deal with the problem of the non-emptiness of the $\gamma$-core for the class of oligopoly TU-games. In order to do that, we first show that oligopoly TU-games in $\gamma$-characteristic function form are well-defined and study some of their properties concerning equilibrium outputs. We go step further by adopting a more general approach in which we assume that any coalition structure can occur. In particular, the coalition structures in which every coalition faces outsiders acting individually can form. For every coalition structure, we construct a normal form oligopoly game for which a Nash equilibrium represents the aggregated equilibrium outputs of the embedded coalitions. Our first result proves that there exists a unique Nash equilibrium for every coalition structure. This permits to conclude that oligopoly TU-games in $\gamma$-characteristic function form are well-defined. Our second result shows that the equilibrium total production is decreasing with the coarseness of the coalition structure. This feature follows from two phenomena. Firstly, when some coalitions merge, the production of the new entity decreases, and secondly, the other coalitions respond by increasing their outputs.

Then, by using these preliminaries results we study the non-emptiness of the $\gamma$-core. We consider two approaches. The first shows that if the inverse demand function is differentiable and if every individual profit function is continuous and concave on the set of strategy profiles, the corresponding oligopoly TU-game is balanced, and therefore has a non-empty $\gamma$-core. This result extends Zhao's core existence result (1999b) for the class of oligopoly TU-games since the $\gamma$-core is included in the $\beta$-core and the $\alpha$-core. A drawback with this approach is that it does not point out any allocation rule belonging to the $\gamma$-core. The second approach provides a new single-valued allocation rule in the $\gamma$-core, called NP(Nash Pro rata)-value, on the class of oligopoly TU-games with linear cost functions and asymmetric capacity constraints. The NP-value distributes to every player the worth of the grand coalition in proportion to his Nash individual output. This result generalizes Funaki and Yamato's core existence result (1999) from no capacity constraint to asymmetric capacity constraints insofar as our oligopoly game also describes a common pool game (Moulin and Watts 1997). We characterize this solution by means of four properties: efficiency, null firm, monotonicity and non-cooperative fairness. Efficiency requires that a solution distributes the worth of the grand coalition among players. The null firm property stipulates that a firm with no production capacity obtains a zero payoff. Monotonicity specifies that if a firm has a production capacity greater than or equal to the production capacity of another firm, then former's payoff will be greater than or equal to latter's payoff. Non-cooperative fairness requires that a solution distributes to every player a payoff proportionally to his individual profit in the 
finest coalition structure. As far as we know, this is the first result that characterizes a solution belonging to a core for a particular class of oligopoly TU-games. Furthermore, we provide an example in which the oligopoly TU-game in $\gamma$-characteristic function form, where the inverse demand function and cost functions are linear, fails to be superadditive, and so convex. This proves that Norde et al.'s result (2002) can not be extended for oligopoly TU-games in $\gamma$-characteristic function form.

The remainder of the paper is structured as follows. In section 2 we introduce the model and some notations. Section 3 presents some properties of oligopoly TU-games in $\gamma$-characteristic function. Section 4 establishes our first main result: oligopoly TU-games in $\gamma$-characteristic function are balanced if the inverse demand function is differentiable and if every individual profit function is continuous and concave on the set of strategy profiles. Section 5 provides a solution in the $\gamma$-core, the NP-value, for the class of oligopoly TU-games with linear cost functions and asymmetric capacity constraints. Furthermore, we propose an axiomatic characterization of this solution. Section 6 gives some concluding remarks.

\section{The model}

Consider an oligopoly situation $\left(N,\left(w_{i}, C_{i}\right)_{i \in N}, p\right)$ where $N=\{1,2, \ldots, n\}$ is the set of firms where $i$ is a representative element, $w_{i} \geq 0$ denote firm $i$ 's capacity constraint, $C_{i}: \mathbb{R}_{+} \longrightarrow \mathbb{R}_{+}, i \in N$, is firm $i$ 's cost function and $p: \mathbb{R}_{+} \longrightarrow \mathbb{R}_{+}$represents the inverse demand function. Throughout this paper, we assume that:

(a) the inverse demand function $p$ is differentiable, strictly decreasing and concave;

(b) every cost function $C_{i}$ is continuous, strictly increasing and convex.

The normal form oligopoly game $\left(N,\left(X_{i}, \pi_{i}\right)_{i \in N}\right)$ associated with the oligopoly situation $\left(N,\left(w_{i}, C_{i}\right)_{i \in N}, p\right)$ is defined as follows:

1. the set of firms is $N=\{1,2, \ldots, n\}$;

2. for every $i \in N$, the individual strategy set is $X_{i}=\left[0, w_{i}\right] \subset \mathbb{R}_{+}$where $x_{i} \in X_{i}$ represents the quantity produced by firm $i$;

3. the set of strategy profiles is $X_{N}=\prod_{i \in N} X_{i}$ where $x=\left(x_{i}\right)_{i \in N}$ is a representative element of $X_{N}$; for every $i \in N$, the individual profit function $\pi_{i}: X_{N} \longrightarrow \mathbb{R}_{+}$is defined as

$$
\pi_{i}(x)=p(X) x_{i}-C_{i}\left(x_{i}\right)
$$

where $X=\sum_{i \in N} x_{i}$ is the joint production.

Note that firm $i$ 's profit depends on its individual output $x_{i}$ and on the total output of its opponents $\sum_{j \in N \backslash\{i\}} x_{j}$.

As mentioned above, we want to analyze the stability of cartels and the incentive for 
merger in oligopoly situations. To this end, we convert the normal form oligopoly game $\left(N,\left(X_{i}, \pi_{i}\right)_{i \in N}\right)$ into an oligopoly TU-game. Let $\mathcal{P}(N)$ be the power set of $N$ and call a subset $S \in \mathcal{P}(N), S \neq \emptyset$, a coalition. In a general framework, a TU-game $(N, v)$ is a set function $v: \mathcal{P}(N) \longrightarrow \mathbb{R}$ with the convention $v(\emptyset)=0$, which assigns a number $v(S) \in \mathbb{R}$ to every coalition $S \in \mathcal{P}(N)$. This number $v(S)$ is the worth of coalition $S$. For a fixed set of players $N$, we denote by $G^{N}$ the set of TU-games where $v$ is a representative element of $G^{N}$.

In a TU-game $v \in G^{N}$, every player $i \in N$ may receive a payoff $\sigma_{i} \in \mathbb{R}$. A vector $\sigma=\left(\sigma_{1}, \ldots, \sigma_{n}\right)$ is a payoff vector. We say that a payoff vector $\sigma \in \mathbb{R}^{n}$ is acceptable if $\sum_{i \in S} \sigma_{i} \geq v(S)$ for every coalition $S \in \mathcal{P}(N)$, i.e. the payoff vector provides a total payoff to members of coalition $S$ that is at least as great as its worth. We say that a payoff vector $\sigma \in \mathbb{R}^{n}$ is efficient if $\sum_{i \in N} \sigma_{i}=v(N)$, i.e. the payoff vector provides a total payoff to all players that is equal to the worth of the grand coalition $N$. The core $C(v)$ of a TU-game $v \in G^{N}$ is the set of all payoff vectors that are both acceptable and efficient, i.e.

$$
C(v)=\left\{\sigma \in \mathbb{R}^{n}: \forall S \subseteq N, \sum_{i \in S} \sigma_{i} \geq v(S) \text { and } \sum_{i \in N} \sigma_{i}=v(N)\right\}
$$

Given a payoff vector in the core, the grand coalition could form and distribute its worth as payoffs to its members in such a way that no coalition can contest this sharing by breaking off from the grand coalition.

There are two main ways of converting a normal form game into a cooperative game called game in $\alpha$ - and $\beta$-characteristic function form respectively (Aumann 1959): in the first case, the worth of a coalition is obtained by computing the profit which its members can guarantee themselves regardless of what outsiders do; in the second case, the worth of a coalition can be derived by computing the minimal profit such that outsiders can prevent its members from getting more. In order to define the $\alpha$ - and $\beta$-characteristic functions, we denote by $X_{S}=\prod_{i \in S} X_{i}$ the strategy set of coalition $S \in \mathcal{P}(N)$ and $X_{-S}=\prod_{i \notin S} X_{i}$ the set of outsiders' strategy profiles for which $x_{S}=\left(x_{i}\right)_{i \in S}$ and $x_{-S}=\left(x_{i}\right)_{i \notin S}$ are the representative elements respectively. Given the normal form oligopoly TU-game $\left(N,\left(X_{i}, \pi_{i}\right)_{i \in N}\right)$, these functions are defined for every coalition $S \in$ $\mathcal{P}(N)$ as

$$
v_{\alpha}(S)=\max _{x_{S} \in X_{S}} \min _{z_{-S} \in X_{-S}} \sum_{i \in S} \pi_{i}\left(x_{S}, z_{-S}\right)
$$

and

$$
v_{\beta}(S)=\min _{z_{-S} \in X_{-S}} \max _{x_{S} \in X_{S}} \sum_{i \in S} \pi_{i}\left(x_{S}, z_{-S}\right)
$$

respectively. ${ }^{3}$ Since the inverse demand function $p$ is strictly decreasing with the joint production $X$, we deduce from (1) that

\footnotetext{
${ }^{3}$ At this step, we assume that these maximization/minimization problems have optimal solutions. This remark will remain valid for the $\gamma$-characteristic function.
} 


$$
\forall x_{S} \in X_{S}, w_{-S}=\left(w_{i}\right)_{i \notin S} \in \arg \min _{z_{-S} \in X_{-S}} \sum_{i \in S} \pi_{i}\left(x_{S}, z_{-S}\right)
$$

where $w_{i} \in X_{i}$ is the full capacity output of firm $i$. Furthermore, for every $S \in \mathcal{P}(N)$, define $B_{S}: X_{-S} \rightarrow X_{S}$ the best reply correspondence of coalition $S$ as

$$
\forall z_{-S} \in X_{-S}, B_{S}\left(z_{-S}\right)=\arg \max _{x_{S} \in X_{S}} \sum_{i \in S} \pi_{i}\left(x_{S}, z_{-S}\right)
$$

For notational convenience, we denote by $x_{S}^{*}\left(z_{-S}\right)$ a representative element of $B_{S}\left(z_{-S}\right)$. Zhao (1999b) shows that the $\alpha$ - and $\beta$-characteristic functions are equal for the class of oligopoly TU-games. This result, (4), (5) and (6) together imply

$$
v_{\alpha}(S)=v_{\beta}(S)=\sum_{i \in S} \pi_{i}\left(x_{S}^{*}\left(w_{-S}\right), w_{-S}\right)
$$

By assuming that every individual profit function $\pi_{i}$ is concave on the set of strategy profiles, the non-emptiness of the $\alpha$ - and $\beta$-cores follows from Zhao's theorem (1999b).

The resorting to the $\alpha$ - and $\beta$-characteristic functions to construct oligopoly TUgames can be questioned insofar as the reaction of external firms to minimize the worth of a deviating coalition by increasing their output at full capacity probably implies substantial damages upon themselves. As in Chander and Tulkens (1997), we consider the alternative blocking rule for which outsiders choose their strategy individually as a best reply facing the deviating coalition. This leads to consider the $\gamma$-characteristic function. In order to define the $\gamma$-characteristic function, for every deviating coalition $S \in \mathcal{P}(N)$ and for any $x_{S} \in X_{S}$, we denote by $\tilde{z}_{-S}\left(x_{S}\right)=\left(\tilde{z}_{i}\left(x_{S}, \tilde{z}_{-S \cup i}\right)\right)_{i \notin S} \in \prod_{i \notin S} B_{i}\left(x_{S}, \tilde{z}_{-S \cup i}\right)$ outsiders' individual best reply strategy profile where $S \cup i$ stands for $S \cup\{i\}$. Given the normal form oligopoly game $\left(N,\left(X_{i}, \pi_{i}\right)_{i \in N}\right)$, the $\gamma$-characteristic function is defined for every coalition $S \in \mathcal{P}(N)$ as

$$
v_{\gamma}(S)=\sum_{i \in S} \pi_{i}\left(x_{S}^{*}\left(\tilde{z}_{-S}\right), \tilde{z}_{-S}\left(x_{S}^{*}\right)\right)
$$

The strategy profile $\left(x_{S}^{*}\left(\tilde{z}_{-S}\right), \tilde{z}_{-S}\left(x_{S}^{*}\right)\right) \in X_{N}$ is called a partial agreement equilibrium under $S$. For a fixed set of players $N$, we denote by $G_{o}^{N} \subseteq G^{N}$ the set of oligopoly TU-games. The following proposition shows that the $\gamma$-core is included in the $\beta$-core, and therefore by (7) in the $\alpha$-core.

Proposition 2.1 Let $v_{\gamma} \in G_{o}^{N}$ and $v_{\beta} \in G_{o}^{N}$ be oligopoly TU-games associated with the oligopoly situation $\left(N,\left(w_{i}, C_{i}\right)_{i \in N}, p\right)$. Then

$$
C\left(v_{\gamma}\right) \subseteq C\left(v_{\beta}\right)
$$

Proof: By (2), we have to show that $v_{\gamma}(S) \geq v_{\beta}(S)$ for every $S \in \mathcal{P}(N) \backslash\{N\}$ and $v_{\gamma}(N)=v_{\beta}(N)$. Clearly, 


$$
\begin{aligned}
v_{\gamma}(N) & =\max _{x \in X_{N}} \sum_{i \in N} \pi_{i}(x) \\
& =v_{\beta}(N) .
\end{aligned}
$$

Moreover, for every $S \in \mathcal{P}(N) \backslash\{N\}$, it holds that

$$
\begin{aligned}
v_{\gamma}(S) & =\sum_{i \in S} \pi_{i}\left(x_{S}^{*}\left(\tilde{z}_{-S}\right), \tilde{z}_{-S}\left(x_{S}^{*}\right)\right) \\
& =\max _{x_{S} \in X_{S}} \sum_{i \in S} \pi_{i}\left(x_{S}, \tilde{z}_{-S}\left(x_{S}^{*}\right)\right) \\
& \geq \max _{x_{S} \in X_{S}} \sum_{i \in S} \pi_{i}\left(x_{S}, w_{-S}\right) \\
& =\min _{z_{-S} \in X_{-S}} \max _{x_{S} \in X_{S}} \sum_{i \in S} \pi_{i}\left(x_{S}, z_{-S}\right) \\
& =v_{\beta}(S)
\end{aligned}
$$

where the inequality and the third equality follow from (5).

\section{Properties of Cournot oligopoly TU-games}

In this section, we study the existence of partial agreement equilibria for every normal form oligopoly game. This will permit us to make sure that the associated oligopoly TUgame in $\gamma$-characteristic function form as in (8) is well-defined, i.e. there exists a unique worth, $v_{\gamma}(S)$, for every coalition $S \in \mathcal{P}(N)$. Moreover, for every $S, T \in \mathcal{P}(N)$ such that $S \subseteq T$, we analyse the variations in equilibrium outputs from a partial agreement equilibrium under $S$ to a partial agreement equilibrium under $T$. To this end, we adopt a more general approach in which we assume that any coalition structure can occur. For every normal form oligopoly game and any coalition structure, we construct an associated normal form oligopoly game for which a Nash equilibrium represents the aggregated equilibrium outputs of the coalitions embedded in the coalition structure.

A coalition structure $\mathcal{P}$ is a partition of the set of firms $N$, i.e. $\mathcal{P}=\left\{S_{1}, \ldots, S_{k}\right\}$, $k \in\{1, \ldots, n\}$. An element of a coalition structure, $S \in \mathcal{P}$, is called an admissible coalition in $\mathcal{P}$. We denote by $\Pi(N)$ the set of coalition structures.

Given the normal form oligopoly game $\left(N,\left(X_{i}, \pi_{i}\right)_{i \in N}\right)$ and the coalition structure $\mathcal{P} \in$ $\Pi(N)$, we say that a strategy profile $\hat{x} \in X_{N}$ is an equilibrium under $\mathcal{P}$ if

$$
\forall S \in \mathcal{P}, \hat{x}_{S} \in B_{S}\left(\hat{x}_{-S}\right)
$$

where $B_{S}$ is the best reply correspondence given by (6). Thus, a partial agreement equilibrium under $S \in \mathcal{P}(N)$ corresponds to an equilibrium under the particular coalition structure denoted by $\mathcal{P}^{S}=\{S\} \cup\{\{i\}: i \notin S\}$. 
For every normal form oligopoly game, we deal with the problem of the existence of an equilibrium under every coalition structure. To this end, given a normal form oligopoly game $\left(N,\left(X_{i}, \pi_{i}\right)_{i \in N}\right)$ and a coalition structure $\mathcal{P} \in \Pi(N)$, we define the normal form oligopoly game $\left(\mathcal{P},\left(X^{S}, \pi_{S}\right)_{S \in \mathcal{P}}\right)$ as follows:

1. the set of players (or admissible coalitions) is $\mathcal{P}$;

2. for every $S \in \mathcal{P}$, the coalition strategy set is $X^{S}=\left[0, w^{S}\right] \subset \mathbb{R}_{+}, w^{S}=$ $\sum_{i \in S} w_{i}$, for which $x^{S}=\sum_{i \in S} x_{i} \in X^{S}$ is a representative element that represents the quantity produced by coalition $S$;

3. the set of strategy profiles is $X^{\mathcal{P}}=\prod_{S \in \mathcal{P}} X^{S}$ for which $x^{\mathcal{P}}=\left(x^{S}\right)_{S \in \mathcal{P}}$ is a representative element; for every $S \in \mathcal{P}$, the coalition cost function $C_{S}: X^{S} \longrightarrow$ $\mathbb{R}_{+}$is defined as

$$
C_{S}\left(x^{S}\right)=\min _{x_{S} \in I\left(x^{S}\right)} \sum_{i \in S} C_{i}\left(x_{i}\right)
$$

where $I\left(x^{S}\right)=\left\{x_{S} \in X_{S}: \sum_{i \in S} x_{i}=x^{S}\right\}$ is the set of strategies of coalition $S$ that permit it to produce the quantity $x^{S}$; for every $S \in \mathcal{P}$, the coalition profit function $\pi_{S}: X^{\mathcal{P}} \longrightarrow \mathbb{R}$ is defined as

$$
\pi_{S}\left(x^{\mathcal{P}}\right)=p(X) x^{S}-C_{S}\left(x^{S}\right)
$$

In order to define the best reply correspondence of the players (the admissible coalitions), for every $S \in \mathcal{P}$, we denote by $X^{-S}=X^{\mathcal{P} \backslash\{S\}}$ the set of outsiders' strategy profiles for which $x^{-S}=x^{\mathcal{P} \backslash\{S\}}$ is a representative element. For every $S \in \mathcal{P}$, define $B^{S}$ : $X^{-S} \rightarrow X^{S}$ the best reply correspondence of coalition $S$ as

$$
\forall z^{-S} \in X^{-S}, B^{S}\left(z^{-S}\right)=\arg \max _{x^{S} \in X^{S}} \pi_{S}\left(x^{S}, z^{-S}\right)
$$

Given the normal form oligopoly game $\left(\mathcal{P},\left(X^{S}, \pi_{S}\right)_{S \in \mathcal{P}}\right)$, we say that a strategy profile $\hat{x}^{\mathcal{P}} \in X^{\mathcal{P}}$ is a Nash equilibrium if

$$
\forall S \in \mathcal{P}, \hat{x}^{S} \in B^{S}\left(\hat{x}^{-S}\right)
$$

where $B^{S}$ is the best reply correspondence given by (13).

The following proposition establishes the existence of an equilibrium under every coalition structure $\mathcal{P} \in \Pi(N)$.

Proposition 3.1 Let $\left(N,\left(X_{i}, \pi_{i}\right)_{i \in N}\right)$ be a normal form oligopoly game. Then, for any coalition structure $\mathcal{P} \in \Pi(N)$, there exists an equilibrium under $\mathcal{P}$. 
Proof: Consider the normal form oligopoly game $\left(\mathcal{P},\left(X^{S}, \pi_{S}\right)_{S \in \mathcal{P}}\right)$ associated with the normal form oligopoly game $\left(N,\left(X_{i}, \pi_{i}\right)_{i \in N}\right)$ and the coalition structure $\mathcal{P} \in \Pi(N)$. We proceed in two parts.

First, we show that there exists a strategy profile $\hat{x}^{\mathcal{P}} \in X^{\mathcal{P}}$ that is a Nash equilibrium for the normal form oligopoly game $\left(\mathcal{P},\left(X^{S}, \pi_{S}\right)_{S \in \mathcal{P}}\right)$ if and only if there exists a strategy profile $\hat{x} \in X_{N}$ that is an equilibrium under $\mathcal{P}$ for the normal form oligopoly game $\left(N,\left(X_{i}, \pi_{i}\right)_{i \in N}\right)$ where $\hat{x}_{S} \in I\left(\hat{x}^{S}\right)$ for every $S \in \mathcal{P}$.

$[\Longrightarrow]$ : let $\hat{x}^{\mathcal{P}} \in X^{\mathcal{P}}$ be a Nash equilibrium for the normal form oligopoly game $\left(\mathcal{P},\left(X^{S}, \pi_{S}\right)_{S \in \mathcal{P}}\right)$.

By (11), for every $S \in \mathcal{P}$ there exists $\hat{x}_{S} \in X_{S}$ such that

$$
\sum_{i \in S} \hat{x}_{i}=\hat{x}^{S} \text { and } \sum_{i \in S} C_{i}\left(\hat{x}_{i}\right)=C_{S}\left(\hat{x}^{S}\right)
$$

For the sake of contradiction assume that the strategy profile $\hat{x}=\left(\hat{x}_{S}\right)_{S \in \mathcal{P}} \in X_{N}$ as in (15) is not an equilibrium under $\mathcal{P}$ for the normal form oligopoly game $\left(N,\left(X_{i}, \pi_{i}\right)_{i \in N}\right)$. It follows that $\hat{x}_{S} \notin B_{S}\left(\hat{x}_{-S}\right)$ for some $S \in \mathcal{P}$, i.e. there exists $\check{x}_{S} \in X_{S}$ such that

$$
\sum_{i \in S} \pi_{i}\left(\hat{x}_{S}, \hat{x}_{-S}\right)<\sum_{i \in S} \pi_{i}\left(\check{x}_{S}, \hat{x}_{-S}\right)
$$

In the normal form oligopoly game $\left(\mathcal{P},\left(X^{S}, \pi_{S}\right)_{S \in \mathcal{P}}\right)$, we denote by $\check{x}^{S} \in X^{S}$ the corresponding strategy of coalition $S$ such that $\check{x}^{S}=\sum_{i \in S} \check{x}_{i}$. By (15), (16) and (11), it holds that

$$
\begin{aligned}
\pi_{S}\left(\hat{x}^{\mathcal{P}}\right) & =p(\hat{X}) \hat{x}^{S}-C_{S}\left(\hat{x}^{S}\right) \\
& =p(\hat{X}) \sum_{i \in S} \hat{x}_{i}-\sum_{i \in S} C_{i}\left(\hat{x}_{i}\right) \\
& =\sum_{i \in S} \pi_{i}\left(\hat{x}_{S}, \hat{x}_{-S}\right) \\
& <\sum_{i \in S} \pi_{i}\left(\check{x}_{S}, \hat{x}_{-S}\right) \\
& =p\left(\sum_{i \in S} \check{x}_{i}+\sum_{i \notin S} \hat{x}_{i}\right) \sum_{i \in S} \check{x}_{i}-\sum_{i \in S} C_{i}\left(\check{x}_{i}\right) \\
& \leq p\left(\check{x}^{S}+\hat{X}-\hat{x}^{S}\right) \check{x}^{S}-C_{S}\left(\check{x}^{S}\right) \\
& =\pi_{S}\left(\check{x}^{S}, \hat{x}^{-S}\right),
\end{aligned}
$$

a contradiction with $\hat{x}^{S} \in B^{S}\left(\hat{x}^{-S}\right)$ for every $S \in \mathcal{P}$.

$[\Longleftarrow]$ : let $\hat{x} \in X_{N}$ be an equilibrium under $\mathcal{P}$ for the normal form oligopoly game $\left(N,\left(X_{i}, \pi_{i}\right)_{i \in N}\right)$. For the associated normal form oligopoly game $\left(\mathcal{P},\left(X^{S}, \pi_{S}\right)_{S \in \mathcal{P}}\right)$, we define the strategy profile $\hat{x}^{\mathcal{P}} \in X^{\mathcal{P}}$ such that

$$
\forall S \in \mathcal{P}, \hat{x}^{S}=\sum_{i \in S} \hat{x}_{i}
$$


From the fact that $\hat{x}_{S} \in I\left(\hat{x}^{S}\right)$ and $\hat{x}_{S} \in B_{S}\left(\hat{x}_{-S}\right)$ we deduce that $\sum_{i \in S} C_{i}\left(\hat{x}_{i}\right)=$ $C_{S}\left(\hat{x}^{S}\right)$ for every $S \in \mathcal{P}$. For the sake of contradiction assume that the strategy profile $\hat{x}^{\mathcal{P}}=\left(\hat{x}^{S}\right)_{S \in \mathcal{P}} \in X^{\mathcal{P}}$ as in (17) is not a Nash equilibrium for the normal form oligopoly game $\left(\mathcal{P},\left(X^{S}, \pi_{S}\right)_{S \in \mathcal{P}}\right)$. It follows that $\hat{x}^{S} \notin B^{S}\left(\hat{x}^{-S}\right)$ for some $S \in \mathcal{P}$, i.e. there exists $\check{x}^{S} \in X^{S}$ such that

$$
\pi_{S}\left(\hat{x}^{S}, \hat{x}^{-S}\right)<\pi_{S}\left(\check{x}^{S}, \hat{x}^{-S}\right)
$$

By (11), we know that there exists $\check{x}_{S} \in X_{S}$ such that

$$
\sum_{i \in S} \check{x}_{i}=\check{x}^{S} \text { and } \sum_{i \in S} C_{i}\left(\check{x}_{i}\right)=C_{S}\left(\check{x}^{S}\right)
$$

By (18) and (19), it holds that

$$
\begin{aligned}
\sum_{i \in S} \pi_{i}(\hat{x}) & =p(\hat{X}) \sum_{i \in S} \hat{x}_{i}-\sum_{i \in S} C_{i}\left(\hat{x}_{i}\right) \\
& =p(\hat{X}) \hat{x}^{S}-C_{S}\left(\hat{x}^{S}\right) \\
& =\pi_{S}\left(\hat{x}^{S}, \hat{x}^{-S}\right) \\
& <\pi_{S}\left(\check{x}^{S}, \hat{x}^{-S}\right) \\
& =p\left(\check{x}^{S}+\hat{X}-\hat{x}^{S}\right) \check{x}^{S}-C_{S}\left(\check{x}^{S}\right) \\
& =p\left(\sum_{i \in S} \check{x}_{i}+\sum_{i \notin S} \hat{x}_{i}\right) \sum_{i \in S} \check{x}_{i}-\sum_{i \in S} C_{i}\left(\check{x}_{i}\right) \\
& =\sum_{i \in S} \pi_{i}\left(\check{x}_{S}, \hat{x}_{-S}\right),
\end{aligned}
$$

a contradiction with $\hat{x}_{S} \in B_{S}\left(\hat{x}_{-S}\right)$ for every $S \in \mathcal{P}$.

Then, we show that the normal form oligopoly game $\left(\mathcal{P},\left(X^{S}, \pi_{S}\right)_{S \in \mathcal{P}}\right)$ admits a unique Nash equilibrium. For every $S \in \mathcal{P}, X^{S}$ is compact and convex and $C_{S}$ as in (11) is continuous, strictly increasing and convex. ${ }^{4}$ Moreover, the inverse demand function $p$ is differentiable, strictly decreasing and concave. It follows from theorem 3.3.3 (page 30) in Okuguchi and Szidarovszky (1990) that there exists a unique Nash equilibrium for the normal form oligopoly game $\left(\mathcal{P},\left(X^{S}, \pi_{S}\right)_{S \in \mathcal{P}}\right)$. From the first part of the proof, we conclude that there exists an equilibrium under $\mathcal{P}$ for the normal form oligopoly game $\left(N,\left(X_{i}, \pi_{i}\right)_{i \in N}\right)$.

Given the normal form oligopoly game $\left(N,\left(X_{i}, \pi_{i}\right)_{i \in N}\right)$, we saw that a partial agreement equilibrium under $S$ corresponds to an equilibrium under $\mathcal{P}^{S}=\{S\} \cup\{\{i\}: i \notin S\}$. Hence, we deduce from proposition 3.1 the following corollary.

\footnotetext{
${ }^{4}$ The properties of the coalition cost function $C_{S}$ follow from the continuity, the strict monotonicity and the convexity of every cost function $C_{i}$.
} 
Corollary 3.2 Let $\left(N,\left(X_{i}, \pi_{i}\right)_{i \in N}\right)$ be a normal form oligopoly game. Then, for every $S \in \mathcal{P}(N)$ it holds that

(i) there exists a partial agreement equilibrium under $S$.

(ii) for any two partial agreement equilibria $\left(x_{S}^{*}\left(\tilde{z}_{-S}\right), \tilde{z}_{-S}\left(x_{S}^{*}\right)\right),\left(y_{S}^{*}\left(\tilde{t}_{-S}\right), \tilde{t}_{-S}\left(y_{S}^{*}\right)\right) \in$ $X_{N}$, it holds that

$$
\sum_{i \in S} x_{S, i}^{*}\left(\tilde{z}_{-S}\right)=\sum_{i \in S} y_{S, i}^{*}\left(\tilde{t}_{-S}\right) \text { and } \sum_{i \in S} C_{i}\left(x_{S, i}^{*}\left(\tilde{z}_{-S}\right)\right)=\sum_{i \in S} C_{i}\left(y_{S, i}^{*}\left(\tilde{t}_{-S}\right)\right)
$$

Point (i) is a direct consequence of proposition 3.1. Point (ii) follows from the uniqueness of the Nash equilibrium in the normal form oligopoly game $\left(\mathcal{P},\left(X^{S}, \pi_{S}\right)_{S \in \mathcal{P}}\right)$. This stems from the fact that members of $S$ can reallocate the total production among themselves. By (8), this implies that the worth of a coalition $S \in \mathcal{P}(N), v_{\gamma}(S)$, is unique. ${ }^{5}$

The following example shows that the continuity of the inverse demand function is not a sufficient condition to guarantee the uniqueness of the worth, $v_{\gamma}(S)$, of every coalition $S \in \mathcal{P}(N)$. This explains why we assumed the differentiability of the inverse demand function.

\section{Example 3.3}

Consider the oligopoly TU-game $v_{\gamma} \in G_{o}^{N}$ associated with the oligopoly situation ${ }^{6}$ $\left(N,\left(w_{i}, C_{i}\right)_{i \in N}, p\right)$ where $N=\{1,2,3\}, w_{1}=2, w_{2}=1, w_{3}=2, C_{1}\left(x_{1}\right)=97 x_{1}$, $C_{2}\left(x_{2}\right)=98 x_{2}, C_{3}\left(x_{3}\right)=98 x_{3}$, and the inverse demand function is defined as

$$
p(X)= \begin{cases}103-X & \text { if } 0 \leq X \leq 3 \\ 50(5-X) & \text { if } 3<X \leq 5\end{cases}
$$

Clearly, $p$ is continuous, piecewise linear and concave but it is not differentiable. Assume that coalition $\{2,3\}$ forms. We show that every strategy profile $x \in X_{N}$ such that (i) $X=3$ and (ii) $x_{2}+x_{3} \in[1 / 25,2]$ is a partial agreement equilibrium under $\{2,3\}$. By (i) we have

$$
\pi_{1}(x)=3 x_{1}
$$

and

$$
\pi_{2}(x)+\pi_{3}(x)=2\left(x_{2}+x_{3}\right) .
$$

\footnotetext{
${ }^{5}$ Point (ii) of corollary 3.2 remains valid for any coalition structure $\mathcal{P} \in \Pi(N)$, i.e. for any two equilibria under $\mathcal{P}, \hat{x}, \check{x} \in X_{N}$, it holds that

$$
\forall S \in \mathcal{P}, \hat{x}^{S}=\check{x}^{S} \text { and } \sum_{i \in S} C_{i}\left(\hat{x}_{i}\right)=\sum_{i \in S} C_{i}\left(\check{x}_{i}\right) .
$$

${ }^{6}$ This oligopoly situation is taken from Norde et al. (2002).
} 
If player 1 increases his output by $\left.\epsilon \in] 0,2-x_{1}\right]$, his new payoff will be

$$
\pi_{1}\left(x_{1}+\epsilon, x_{2}, x_{3}\right)=(3-50 \epsilon)\left(x_{1}+\epsilon\right)
$$

Conversely, if he decides to decrease his output by $\left.\delta \in] 0, x_{1}\right]$, he will obtain

$$
\pi_{1}\left(x_{1}-\delta, x_{2}, x_{3}\right)=(3+\delta)\left(x_{1}-\delta\right)
$$

Similarly, if coalition $\{2,3\}$ increases its output by $\left.\left.\epsilon+\epsilon^{\prime} \in\right] 0,3-x_{2}-x_{3}\right]$ where $\epsilon \in$ $\left[0,1-x_{2}\right]$ and $\epsilon^{\prime} \in\left[0,2-x_{3}\right]$, its new payoff will be

$$
\sum_{i=2}^{3} \pi_{i}\left(x_{1}, x_{2}+\epsilon, x_{3}+\epsilon^{\prime}\right)=\left(2-50\left(\epsilon+\epsilon^{\prime}\right)\right)\left(x_{2}+x_{3}+\epsilon+\epsilon^{\prime}\right)
$$

On the contrary, if it decreases its output by $\left.\left.\delta+\delta^{\prime} \in\right] 0, x_{2}+x_{3}\right]$ with $\delta \in\left[0, x_{2}\right]$ and $\delta^{\prime} \in\left[0, x_{3}\right]$, it will obtain

$$
\sum_{i=2}^{3} \pi_{i}\left(x_{1}, x_{2}-\delta, x_{3}-\delta^{\prime}\right)=\left(2+\delta+\delta^{\prime}\right)\left(x_{2}+x_{3}-\delta-\delta^{\prime}\right)
$$

In all cases (20), (21) (22) and (23), given (ii), neither player 1 nor coalition $\{2,3\}$ can improve their payoff. We conclude that every strategy profile $x \in X_{N}$ satisfying (i) and (ii) is a partial agreement equilibrium under $\{2,3\}$. Thus, the worth of coalition $\{2,3\}$ belongs to $[2 / 25,4]$, so it is not unique.

Now, for every normal form oligopoly game $\left(N,\left(X_{i}, \pi_{i}\right)_{i \in N}\right)$ and every coalition $S \in$ $\mathcal{P}(N)$, we study the variations of equilibrium outputs of $S$ according to the coarseness of the coalition structure in which it is embedded. To this end, we introduce a binary relation $\leq^{F}$ on $\Pi(N)$ defined as follows: we say that a coalition structure $\mathcal{P} \in \Pi(N)$ is finer than a coalition structure $\mathcal{P}^{\prime} \in \Pi(N)$ (or $\mathcal{P}^{\prime}$ is coarser than $\mathcal{P}$ ) which we write $\mathcal{P}^{\prime} \leq F \mathcal{P}$ if for every admissible coalition $S$ in $\mathcal{P}$ there exists an admissible coalition $T$ in $\mathcal{P}^{\prime}$ such that $T \supseteq S$. Note that $\left(\Pi(N), \leq^{F}\right)$ is a complete lattice.

Given the normal form oligopoly game $\left(\mathcal{P},\left(X^{S}, \pi_{S}\right)_{S \in \mathcal{P}}\right)$, in order to define some properties of the best reply correspondence $B^{S}$ given by (13) of every coalition $S \in \mathcal{P}$, we introduce the notions of forward and backward divided differences. For any function $f: \mathbb{R}_{+} \longrightarrow \mathbb{R}$ and all $\epsilon>0$, define the forward and backward divided differences $f^{+}: \mathbb{R}_{+} \times \mathbb{R}_{+} \longrightarrow \mathbb{R}$ and $f^{-}: \mathbb{R}_{+} \times \mathbb{R}_{+} \longrightarrow \mathbb{R}$ as

$$
f^{+}(a, \epsilon)=\frac{1}{\epsilon}(f(a+\epsilon)-f(a))
$$

and

$$
f^{-}(a, \epsilon)=\frac{1}{\epsilon}(f(a)-f(a-\epsilon)) .
$$

For every admissible coalition $S$ in $\mathcal{P}$ and all $\epsilon>0$, define the functions $\varphi_{S}^{+}: X^{S} \times$ $X^{-S} \times \mathbb{R}_{+} \longrightarrow \mathbb{R}$ and $\varphi_{S}^{-}: X^{S} \times X^{-S} \times \mathbb{R}_{+} \longrightarrow \mathbb{R}$ as 


$$
\varphi_{S}^{+}\left(x^{S}, x^{-S}, \epsilon\right)=p(X+\epsilon)+x^{S} p^{+}(X, \epsilon)-C_{S}^{+}\left(x^{S}, \epsilon\right)
$$

and

$$
\varphi_{S}^{-}\left(x^{S}, x^{-S}, \epsilon\right)=p(X-\epsilon)+x^{S} p^{-}(X, \epsilon)-C_{S}^{-}\left(x^{S}, \epsilon\right) .
$$

We see that an admissible coalition $S \in \mathcal{P}$ can't benefit from increasing its output by $\epsilon$ if and only if $\varphi_{S}^{+}\left(x^{S}, x^{-S}, \epsilon\right) \leq 0$; it can't benefit from decreasing its output by $\epsilon$ if and only if $\varphi_{S}^{-}\left(x^{S}, x^{-S}, \epsilon\right) \geq 0$. Thus, the best reply correspondence $B^{S}$ of every admissible coalition $S \in \mathcal{P}$ given by (13) has the three following properties:

1. $0 \in B^{S}\left(x^{-S}\right) \Longleftrightarrow \varphi_{S}^{+}\left(0, x^{-S}, \epsilon\right) \leq 0, \forall \epsilon>0$;

2. $w^{S} \in B^{S}\left(x^{-S}\right) \Longleftrightarrow \varphi_{S}^{-}\left(w^{S}, x^{-S}, \epsilon\right) \geq 0, \forall \epsilon>0$;

3. $\left.x^{S} \in B^{S}\left(x^{-S}\right): x^{S} \in\right] 0, w^{S}\left[\Longleftrightarrow \varphi_{S}^{-}\left(x^{S}, x^{-S}, \epsilon\right) \geq 0 \geq \varphi_{S}^{+}\left(x^{S}, x^{-S}, \epsilon^{\prime}\right)\right.$, $\forall \epsilon, \epsilon^{\prime}>0$.

The following proposition compares equilibria under $\mathcal{P}$ and $\mathcal{P}^{\prime}$ such that $\mathcal{P}^{\prime} \leq F \mathcal{P}$.

Proposition 3.4 Let $\mathcal{P}, \mathcal{P}^{\prime} \in \Pi(N)$ be two coalition structures such that $\mathcal{P}^{\prime} \leq F \mathcal{P}$. Let $\hat{x}^{\mathcal{P}} \in X^{\mathcal{P}}$ and $\check{x}^{\mathcal{P}^{\prime}} \in X^{\mathcal{P}^{\prime}}$ be the Nash equilibria of the normal form oligopoly games $\left(\mathcal{P},\left(X^{S}, \pi_{S}\right)_{S \in \mathcal{P}}\right)$ and $\left(\mathcal{P}^{\prime},\left(X^{S}, \pi_{S}\right)_{S \in \mathcal{P}^{\prime}}\right)$ respectively. Then, it holds that

(i) $\check{X} \leq \hat{X}$

(ii) $\forall(S, T) \in \mathcal{P} \times \mathcal{P}^{\prime}$ such that $S \subseteq T, \hat{x}^{S} \leq \check{x}^{T}$

(iii) $\sum_{T \in \mathcal{P}^{\prime} \backslash \mathcal{P}} \check{x}^{T} \leq \sum_{S \in \mathcal{P} \backslash \mathcal{P}^{\prime}} \hat{x}^{S}$

In order to establish the proof of proposition 3.4, we first need the following lemmas.

Lemma 3.5 Let $\mathcal{P}, \mathcal{P}^{\prime} \in \Pi(N)$ be two coalition structures for which there exist $T \in \mathcal{P}^{\prime}$ and $S_{l} \in \mathcal{P}, l \in\{1, \ldots, p\}, p \in\{1, \ldots, n\}$, such that $T=\bigcup_{l=1}^{p} S_{l}$. Let $\hat{x}^{\mathcal{P}} \in X^{\mathcal{P}}$ and $\check{x}^{\mathcal{P}^{\prime}} \in X^{\mathcal{P}^{\prime}}$ be the Nash equilibria of the normal form oligopoly games $\left(\mathcal{P},\left(X^{S}, \pi_{S}\right)_{S \in \mathcal{P}}\right)$ and $\left(\mathcal{P}^{\prime},\left(X^{S}, \pi_{S}\right)_{S \in \mathcal{P}^{\prime}}\right)$ respectively. If $\hat{X} \leq \check{X}$ then it holds that $\check{x}^{T} \leq \sum_{l=1}^{p} \hat{x}^{S_{l}}$.

Proof: Let $\mathcal{P}, \mathcal{P}^{\prime} \in \Pi(N)$ be two coalition structures satisfying the conditions of lemma 3.5. For all $l \in\{1, \ldots, p\}$ denote by $\check{x}^{S_{l}}$ the output of subset $S_{l}$ in coalition $T$ so that $\sum_{l=1}^{p} \check{x}^{S_{l}}=\check{x}^{T}$. By the definition of a Nash equilibrium, for all $\epsilon, \epsilon^{\prime}>0$ and all $l \in\{1, \ldots, p\}$, it holds that

$$
\varphi_{S_{l}}^{+}\left(\hat{x}^{S_{l}}, \hat{x}^{-S_{l}}, \epsilon\right) \leq 0
$$

and

$$
\underbrace{p\left(\check{X}-\epsilon^{\prime}\right)+\check{x}^{T} p^{-}\left(\check{X}, \epsilon^{\prime}\right)-C_{S_{l}}^{-}\left(\check{x}^{S_{l}}, \epsilon^{\prime}\right)}_{A_{l}^{\epsilon^{\prime}}} \geq 0
$$


where the second inequality means that, under the Nash equilibrium $\check{x}^{\mathcal{P}^{\prime}} \in X^{\mathcal{P}^{\prime}}$, neither coalition $T$ nor any of its subset $S_{l}, l \in\{1, \ldots, p\}$, can benefit from decreasing their output. For all $\epsilon, \epsilon^{\prime}>0$ and every $l \in\{1, \ldots, p\}$, define $Q_{\epsilon, \epsilon^{\prime}}^{l}=\varphi_{S_{l}}^{+}\left(\hat{x}^{S_{l}}, \hat{x}^{-S_{l}}, \epsilon\right)-A_{l}^{\epsilon^{\prime}} \leq$ 0 . For the sake of contradiction assume that there exists $l \in\{1, \ldots, p\}$ such that $\check{x}^{S_{l}}>\hat{x}^{S_{l}}$. We have

$$
\begin{aligned}
Q_{\epsilon, \epsilon^{\prime}}^{l}= & p(\hat{X}+\epsilon)-p\left(\check{X}-\epsilon^{\prime}\right) \\
& +\hat{x}^{S_{l}} p^{+}(\hat{X}, \epsilon)-\check{x}^{T} p^{-}\left(\check{X}, \epsilon^{\prime}\right) \\
& +C_{S_{l}}^{-}\left(\check{x}^{S_{l}}, \epsilon^{\prime}\right)-C_{S_{l}}^{+}\left(\hat{x}^{S_{l}}, \epsilon\right) .
\end{aligned}
$$

In order to obtain a contradiction, it is sufficient to show that $Q_{\epsilon, \epsilon^{\prime}}^{l}$ is positive for small enough $\epsilon$ and $\epsilon^{\prime}=\epsilon$. First, take $\epsilon=\epsilon^{\prime}$ and $\epsilon<\check{x}^{S_{l}}-\hat{x}^{S_{l}}$. By the convexity of cost functions and the definitions of $C_{S_{l}}^{+}$and $C_{S_{l}}^{-}$, it holds that

$$
\begin{aligned}
C_{S_{l}}^{+}\left(\hat{x}^{S_{l}}, \epsilon\right) & \leq C_{S_{l}}^{+}\left(\check{x}^{S_{l}}-\epsilon, \epsilon\right) \\
& =C_{S_{l}}^{-}\left(\check{x}^{S_{l}}, \epsilon^{\prime}\right)
\end{aligned}
$$

By (24) and the differentiability of the inverse demand function $p$ it follows that

$$
\begin{aligned}
\lim _{\epsilon \longrightarrow 0} Q_{\epsilon, \epsilon}^{l} & \geq p(\hat{X})-p(\check{X})+\hat{x}^{S_{l}} p^{\prime}(\hat{X})-\check{x}^{T} p^{\prime}(\check{X}) \\
& >0
\end{aligned}
$$

where the strict inequality follows from the fact that the inverse demand function $p$ is strictly decreasing and concave and from the assumption $\hat{x}^{S_{l}}<\check{x}^{S_{l}} \leq \check{x}^{T}$. Hence, we obtain a contradiction with $Q_{\epsilon, \epsilon^{\prime}}^{l}=\varphi_{S_{l}}^{+}\left(\hat{x}^{S_{l}}, \hat{x}^{-S_{l}}, \epsilon\right)-A_{l}^{\epsilon^{\prime}} \leq 0$ for all $\epsilon, \epsilon^{\prime}>0$. So, for all $l \in\{1, \ldots, p\}$ we have $\check{x}^{S_{l}} \leq \hat{x}^{S_{l}}$ which implies $\check{x}^{T}=\sum_{l=1}^{p} \check{x}^{S_{l}} \leq \sum_{l=1}^{p} \hat{x}^{S_{l}}$.

Lemma 3.6 Let $\mathcal{P}, \mathcal{P}^{\prime} \in \Pi(N)$ be two coalition structures for which there exist $T \in \mathcal{P}^{\prime}$ and $S_{l} \in \mathcal{P}, l \in\{1, \ldots, p\}, p \in\{1, \ldots, n\}$, such that $T=\bigcup_{l=1}^{p} S_{l}$. Let $\hat{x}^{\mathcal{P}} \in X^{\mathcal{P}}$ and $\check{x}^{\mathcal{P}^{\prime}} \in X^{\mathcal{P}^{\prime}}$ be the Nash equilibria of the normal form oligopoly games $\left(\mathcal{P},\left(X^{S}, \pi_{S}\right)_{S \in \mathcal{P}}\right)$ and $\left(\mathcal{P}^{\prime},\left(X^{S}, \pi_{S}\right)_{S \in \mathcal{P}^{\prime}}\right)$ respectively. If $\check{X} \leq \hat{X}$ then it holds that $\check{x}^{T} \geq \hat{x}^{S_{l}}$ for all $l \in\{1, \ldots, p\}$.

Proof: Let $\mathcal{P}, \mathcal{P}^{\prime} \in \Pi(N)$ be two coalition structures satisfying the conditions of lemma 3.6. By the definition of a Nash equilibrium, for all $\epsilon, \epsilon^{\prime}>0$ and all $l \in\{1, \ldots, p\}$, it holds that $\varphi_{T}^{+}\left(\check{x}^{T}, \check{x}^{-T}, \epsilon\right) \leq 0$ and $\varphi_{S_{l}}^{-}\left(\hat{x}^{S_{l}}, \hat{x}^{-S_{l}}, \epsilon^{\prime}\right) \geq 0$. For all $\epsilon, \epsilon^{\prime}>0$ and every $l \in\{1, \ldots, p\}$, define $Q_{\epsilon, \epsilon^{\prime}}^{l}=\varphi_{T}^{+}\left(\check{x}^{T}, \check{x}^{-T}, \epsilon\right)-\varphi_{S_{l}}^{-}\left(\hat{x}^{S_{l}}, \hat{x}^{-S_{l}}, \epsilon^{\prime}\right) \leq 0$. For the sake of contradiction assume that there exists $l \in\{1, \ldots, p\}$ such that $\hat{x}^{S_{l}}>\check{x}^{T}$. We have

$$
\begin{aligned}
Q_{\epsilon, \epsilon^{\prime}}^{l}= & p(\check{X}+\epsilon)-p\left(\hat{X}-\epsilon^{\prime}\right) \\
& +\check{x}^{T} p^{+}(\check{X}, \epsilon)-\hat{x}^{S_{l}} p^{-}\left(\hat{X}, \epsilon^{\prime}\right) \\
& +C_{S_{l}}^{-}\left(\hat{x}^{S_{l}}, \epsilon^{\prime}\right)-C_{T}^{+}\left(\check{x}^{T}, \epsilon\right) .
\end{aligned}
$$


As in the previous lemma, it is sufficient to show that $Q_{\epsilon, \epsilon^{\prime}}^{l}$ is positive for small enough $\epsilon$ and $\epsilon^{\prime}=\epsilon$. First, take $\epsilon=\epsilon^{\prime}$ and $\epsilon<\hat{x}^{S_{l}}-\check{x}^{T}$. By the convexity of cost functions, the definitions of $C_{T}^{+}, C_{S_{l}}^{+}$and $C_{S_{l}}^{-}$, and from the fact that it is less costly for coalition $T$ to redistribute an extra cost than for any of its subsets $S_{l}, l \in\{1, \ldots, p\}$, it holds that

$$
\begin{aligned}
C_{T}^{+}\left(\check{x}^{T}, \epsilon\right) & \leq C_{T}^{+}\left(\hat{x}^{S_{l}}-\epsilon, \epsilon\right) \\
& \leq C_{S_{l}}^{+}\left(\hat{x}^{S_{l}}-\epsilon, \epsilon\right) \\
& =C_{S_{l}}^{-}\left(\hat{x}^{S_{l}}, \epsilon^{\prime}\right)
\end{aligned}
$$

By (25) and the differentiability of the inverse demand function $p$ it follows that

$$
\begin{aligned}
\lim _{\epsilon \longrightarrow 0} Q_{\epsilon, \epsilon}^{l} & \geq p(\check{X})-p(\hat{X})+\check{x}^{T} p^{\prime}(\check{X})-\hat{x}^{S_{l}} p^{\prime}(\hat{X}) \\
& >0
\end{aligned}
$$

where the strict inequality follows from the fact that the inverse demand function $p$ is strictly decreasing and concave and from the assumption $\hat{x}^{S_{l}}>\check{x}^{T}$. Hence, we obtain a contradiction with $Q_{\epsilon, \epsilon^{\prime}}^{l}=\varphi_{T}^{+}\left(\check{x}^{T}, \check{x}^{-T}, \epsilon\right)-\varphi_{S_{l}}^{-}\left(\hat{x}^{S_{l}}, \hat{x}^{-S_{l}}, \epsilon^{\prime}\right) \leq 0$ for all $\epsilon, \epsilon^{\prime}>0$. So, for all $l \in\{1, \ldots, p\}$ we have $\check{x}^{T} \geq \hat{x}^{S_{l}}$.

Now, we are ready to establish the proof of proposition 3.4.

Proof: First, in order to prove (i), assume by contradiction that $\hat{X}<\check{X}$. Since $\mathcal{P}^{\prime} \leq F$ $\mathcal{P}$, for every $T \in \mathcal{P}^{\prime}$ there exists $S_{l} \in \mathcal{P}, l \in\{1, \ldots, p\}, p \in\{1, \ldots, n\}$, such that $T=\bigcup_{l=1}^{p} S_{l}$. It follows from lemma 3.5 that for every $T \in \mathcal{P}^{\prime}$ and every $S_{l} \in \mathcal{P}$, $l \in\{1, \ldots, p\}$, we have $\check{x}^{T} \leq \sum_{l=1}^{p} \hat{x}^{S_{l}}$, and so $\check{X} \leq \hat{X}$ a contradiction.

Then, point (ii) follows directly from (i) and lemma 3.6.

Finally, point (iii) is a consequence of points (i) and (ii). Indeed, by (i) it holds that

$$
\begin{aligned}
\check{X} \leq \hat{X} & \Longleftrightarrow \sum_{T \in \mathcal{P}^{\prime}} \check{x}^{T} \leq \sum_{S \in \mathcal{P}} \hat{x}^{S} \\
& \Longleftrightarrow \sum_{T \in \mathcal{P}^{\prime} \backslash \mathcal{P}} \check{x}^{T}+\sum_{T \in \mathcal{P}^{\prime} \cap \mathcal{P}} \check{x}^{T} \leq \sum_{S \in \mathcal{P} \backslash \mathcal{P}^{\prime}} \hat{x}^{S}+\sum_{S \in \mathcal{P} \cap \mathcal{P}^{\prime}} \hat{x}^{S} \\
& \Longleftrightarrow \sum_{T \in \mathcal{P}^{\prime} \cap \mathcal{P}} \check{x}^{T}-\sum_{S \in \mathcal{P} \cap \mathcal{P}^{\prime}} \hat{x}^{S} \leq \sum_{S \in \mathcal{P} \backslash \mathcal{P}^{\prime}} \hat{x}^{S}-\sum_{T \in \mathcal{P}^{\prime} \backslash \mathcal{P}} \check{x}^{T}
\end{aligned}
$$

Moreover, by (ii) it follows that

$$
\sum_{T \in \mathcal{P}^{\prime} \cap \mathcal{P}} \check{x}^{T}-\sum_{S \in \mathcal{P} \cap \mathcal{P}^{\prime}} \hat{x}^{S} \geq 0
$$

Combining (26) and (27) we obtain $\sum_{T \in \mathcal{P}^{\prime} \backslash \mathcal{P}} \check{x}^{T} \leq \sum_{S \in \mathcal{P} \backslash \mathcal{P}^{\prime}} \hat{x}^{S}$. 
Point (iii) of proposition 3.4 can not be improved in the sense that it does not always hold that for every $T \in \mathcal{P}^{\prime}$ and every $S_{l} \in \mathcal{P}, l \in\{1, \ldots, p\}$, such that $T=\bigcup_{l=1}^{p} S_{l}$, we have $\check{x}^{T} \leq \sum_{l=1}^{p} \hat{x}^{S_{l}}$. This is illustrated in the following example.

\section{Example 3.7}

Consider the oligopoly situation $\left(N,\left(w_{i}, C_{i}\right)_{i \in N}, p\right)$ where $N=\{1, \ldots, 8\}, w_{i}=3 / 2$ and $C_{i}\left(x_{i}\right)=x_{i}$ for all $i \in N$, and the inverse demand function is defined as $p(X)=12-X$. Let $\mathcal{P}=\{\{i\}: i \in N\}, \mathcal{P}^{\prime}=\{\{1,2\},\{3,4,5\},\{6,7,8\}\} \in \Pi(N)$ be two coalition structures such that $\mathcal{P}^{\prime} \leq F \mathcal{P}$. The Nash equilibria of the normal form oligopoly games $\left(N,\left(X_{i}, \pi_{i}\right)_{i \in N}\right)$ and $\left(\mathcal{P}^{\prime},\left(X^{S}, \pi_{S}\right)_{S \in \mathcal{P}^{\prime}}\right)$ are given by $\tilde{z}_{i}\left(\tilde{z}_{-i}\right)^{7}=11 / 9$ for all $i \in N$ and by $\check{x}^{\prime}=(11 / 4,11 / 4,11 / 4)$ respectively. So, we have $\check{x}^{\{1,2\}}=11 / 4>22 / 9=$ $\tilde{z}_{1}\left(\tilde{z}_{-1}\right)+\tilde{z}_{2}\left(\tilde{z}_{-2}\right)$.

We saw that the Nash equilibrium outputs of the normal form oligopoly game $\left(\mathcal{P},\left(X^{S}, \pi_{S}\right)_{S \in \mathcal{P}}\right)$ are equal to the aggregated equilibrium outputs under $\mathcal{P}$ of the normal form oligopoly game $\left(N,\left(X_{i}, \pi_{i}\right)_{i \in N}\right)$. For notational convenience, given a normal form oligopoly game $\left(N,\left(X_{i}, \pi_{i}\right)_{i \in N}\right)$, a coalition $S \in \mathcal{P}(N)$, and a partial agreement equilibrium under $S$, $\left(x_{S}^{*}\left(\tilde{z}_{-S}\right), \tilde{z}_{-S}\left(x_{S}^{*}\right)\right) \in X_{N}$, where $\tilde{z}_{-S}\left(x_{S}^{*}\right)$ stands for $\left(\tilde{z}_{i}\left(x_{S}^{*}, \tilde{z}_{-S \cup i}\right)\right)_{i \notin S}$, we denote by $X^{P, S}$ the total output of the partial agreement equilibrium under $S$, i.e.

$$
X^{P, S}=\sum_{i \in S} x_{S, i}^{*}\left(\tilde{z}_{-S}\right)+\sum_{i \notin S} \tilde{z}_{i}\left(x_{S}^{*}, \tilde{z}_{-S \cup i}\right) .
$$

For partial agreement equilibria we deduce from proposition 3.4 the following corollary.

Corollary 3.8 Let $\left(N,\left(X_{i}, \pi_{i}\right)_{i \in N}\right)$ be a normal form oligopoly game. Then, for every $S, T \in \mathcal{P}(N)$ such that $S \subseteq T$, it holds that

(i) $X^{P, S} \geq X^{P, T}$

(ii) $\forall i \notin T, \tilde{z}_{i}\left(x_{S}^{*}, \tilde{z}_{-S \cup i}\right) \leq \tilde{z}_{i}\left(x_{T}^{*}, \tilde{z}_{-T \cup i}\right)$

(iii) $\sum_{i \in S} x_{S, i}^{*}\left(\tilde{z}_{-S}\right) \leq \sum_{i \in T} x_{T, i}^{*}\left(\tilde{z}_{-T}\right)$

(iv) $\sum_{i \in T} x_{T, i}^{*}\left(\tilde{z}_{-T}\right) \leq \sum_{i \in S} x_{S, i}^{*}\left(\tilde{z}_{-S}\right)+\sum_{i \in T \backslash S} \tilde{z}_{i}\left(x_{S}^{*}, \tilde{z}_{-S \cup i}\right)$

Points (ii) and (iii) follow from (ii) of proposition 3.4. Only point (ii) requires a comment: it stipulates that players outside $T$ increase their output from the partial agreement equilibrium under $S$ to the partial agreement equilibrium under $T$.

\footnotetext{
${ }^{7}$ Given the normal form oligopoly game $\left(N,\left(X_{i}, \pi_{i}\right)_{i \in N}\right)$, we denote by $\left(\tilde{z}_{i}\left(\tilde{z}_{-i}\right)\right)_{i \in N} \in X_{N}$ its unique Nash equilibrium. For all $j \in N$, we will also use this notation to refer to every partial agreement equilibrium under $\mathcal{P}^{\{j\}}=\{\{i\}: i \in N\}$.
} 


\section{Balancedness of Cournot oligopoly TU-games}

In this section, we analyze the non-emptiness of the $\gamma$-core. Following the BondarevaShapley theorem (Bondareva 1963, Shapley 1967), balancedness property is a necessary and sufficient condition to guarantee the non-emptiness of the core for every TU-game $v \in G^{N}$. Let $\mathcal{B} \subseteq \mathcal{P}(N)$ be a family of coalitions and denote by $\mathcal{B}_{i}=\{S \in \mathcal{B}: i \in S\}$ the subset of those coalitions of which player $i$ is a member. Then $\mathcal{B}$ is said to be a balanced family of coalitions if for every $S \in \mathcal{B}$ there exists a balancing weight $\lambda_{S} \in \mathbb{R}_{+}$such that $\sum_{S \in \mathcal{B}_{i}} \lambda_{S}=1$ for every $i \in N$. A TU-game $v \in G^{N}$ is balanced if for every balanced collection $\mathcal{B}$ it holds that

$$
\sum_{S \in \mathcal{B}} \lambda_{S} v(S) \leq v(N)
$$

The Bondareva-Shapley theorem is the following.

Theorem 4.1 A TU-game $v \in G^{N}$ has a non-empty core if and only if it is balanced.

The main result of this section states that for any normal form oligopoly game for which every individual profit function is concave on the set of strategy profiles, the associated oligopoly TU-game is balanced, and so admits a non-empty $\gamma$-core.

Theorem 4.2 Let $\left(N,\left(X_{i}, \pi_{i}\right)_{i \in N}\right)$ be a normal form oligopoly game such that $\pi_{i}$ is concave on $X_{N}$ for every $i \in N$. Then the associated oligopoly TU-game $v_{\gamma} \in G_{o}^{N}$ is balanced and therefore has a non-empty $\gamma$-core.

In order to establish the proof of theorem 4.2, we first need the following lemma. Given a normal form oligopoly game $\left(N,\left(X_{i}, \pi_{i}\right)_{i \in N}\right)$, a balanced family of coalitions $\mathcal{B} \subseteq \mathcal{P}(N)$, and every partial agreement equilibrium under $S,\left(x_{S}^{*}\left(\tilde{z}_{-S}\right), \tilde{z}_{-S}\left(x_{S}^{*}\right)\right) \in X_{N}$, such that $S \in \mathcal{B}$, define $y \in X_{N}$ a strategy profile as

$$
\forall i \in N, y_{i}=\sum_{S \in \mathcal{B}_{i}} \lambda_{S} x_{S, i}^{*}\left(\tilde{z}_{-S}\right)
$$

Lemma 4.3 Let $y \in X_{N}$ be a strategy profile as in (28). Then

$$
\forall j \in N, \quad \sum_{S \in \mathcal{B}_{j}} \lambda_{S} X^{P, S} \geq Y
$$

where $Y=\sum_{i \in N} y_{i}$.

Proof: Pick any $j \in N$. First, we show that

$$
\sum_{S \in \mathcal{B}_{j}} \lambda_{S} \sum_{i \notin S} \tilde{z}_{i}\left(x_{S}^{*}, \tilde{z}_{-S \cup i}\right) \geq \sum_{S \in \mathcal{B} \backslash \mathcal{B}_{j}} \lambda_{S} \sum_{i \in S} x_{S, i}^{*}\left(\tilde{z}_{-S}\right)
$$

By (ii) and (iv) of corollary 3.8, it follows that 


$$
\begin{aligned}
\sum_{S \in \mathcal{B}_{j}} \lambda_{S} \sum_{i \notin S} \tilde{z}_{i}\left(x_{S}^{*}, \tilde{z}_{-S \cup i}\right) & \geq \sum_{S \in \mathcal{B}_{j}} \lambda_{S} \sum_{i \notin S} \tilde{z}_{i}\left(\tilde{z}_{-i}\right) \\
& =\sum_{S \in \mathcal{B}_{j}} \lambda_{S}\left(\sum_{i \in N} \tilde{z}_{i}\left(\tilde{z}_{-i}\right)-\sum_{i \in S} \tilde{z}_{i}\left(\tilde{z}_{-i}\right)\right) \\
& =\sum_{i \in N} \tilde{z}_{i}\left(\tilde{z}_{-i}\right)-\sum_{S \in \mathcal{B}_{j}} \lambda_{S} \sum_{i \in S} \tilde{z}_{i}\left(\tilde{z}_{-i}\right) \\
& =\sum_{S \in \mathcal{B}} \lambda_{S} \sum_{i \in S} \tilde{z}_{i}\left(\tilde{z}_{-i}\right)-\sum_{S \in \mathcal{B}_{j}} \lambda_{S} \sum_{i \in S} \tilde{z}_{i}\left(\tilde{z}_{-i}\right) \\
& =\sum_{S \in \mathcal{B} \backslash \mathcal{B}_{j}} \lambda_{S} \sum_{i \in S} \tilde{z}_{i}\left(\tilde{z}_{-i}\right) \\
& \geq \sum_{S \in \mathcal{B} \backslash \mathcal{B}_{j}} \lambda_{S} \sum_{i \in S} x_{S, i}^{*}\left(\tilde{z}_{-S}\right) .
\end{aligned}
$$

Thus, by (29) it holds that

$$
\begin{aligned}
\sum_{S \in \mathcal{B}_{j}} \lambda_{S} X^{P, S} & =\sum_{S \in \mathcal{B}_{j}} \lambda_{S} \sum_{i \in S} x_{S, i}^{*}\left(\tilde{z}_{-S}\right)+\sum_{S \in \mathcal{B}_{j}} \lambda_{S} \sum_{i \notin S} \tilde{z}_{i}\left(x_{S}^{*}, \tilde{z}_{-S \cup i}\right) \\
& \geq \sum_{S \in \mathcal{B}_{j}} \lambda_{S} \sum_{i \in S} x_{S, i}^{*}\left(\tilde{z}_{-S}\right)+\sum_{S \in \mathcal{B} \backslash \mathcal{B}_{j}} \lambda_{S} \sum_{i \in S} x_{S, i}^{*}\left(\tilde{z}_{-S}\right) \\
& =\sum_{S \in \mathcal{B}} \lambda_{S} \sum_{i \in S} x_{S, i}^{*}\left(\tilde{z}_{-S}\right) \\
& =\sum_{i \in N} \sum_{S \in \mathcal{B}_{i}} \lambda_{S} x_{S, i}^{*}\left(\tilde{z}_{-S}\right) \\
& =Y
\end{aligned}
$$

which completes the proof.

Helm (2001) obtains a similar result for games with multilateral environmental externalities. Now, we are ready to establish the proof of theorem 4.2.

Proof: Let $\mathcal{B} \subseteq \mathcal{P}(N)$ be a balanced family of coalitions and $y \in X_{N}$ be a strategy profile as in (28). By the concavity of every individual profit function $\pi_{i}$ on $X_{N}$, lemma 4.3 and the strict monotonicity of the inverse demand function, and the Pareto efficiency of the worth of the grand coalition it holds that 


$$
\begin{aligned}
\sum_{S \in \mathcal{B}} \lambda_{S} v_{\gamma}(S) & =\sum_{S \in \mathcal{B}} \lambda_{S} \sum_{i \in S} \pi_{i}\left(x_{S}^{*}\left(\tilde{z}_{-S}\right), \tilde{z}_{-S}\left(x_{S}^{*}\right)\right) \\
& =\sum_{i \in N} \sum_{S \in \mathcal{B}_{i}} \lambda_{S} \pi_{i}\left(x_{S}^{*}\left(\tilde{z}_{-S}\right), \tilde{z}_{-S}\left(x_{S}^{*}\right)\right) \\
& \leq \sum_{i \in N} \pi_{i}\left(\sum_{S \in \mathcal{B}_{i}} \lambda_{S}\left(x_{S}^{*}\left(\tilde{z}_{-S}\right), \tilde{z}_{-S}\left(x_{S}^{*}\right)\right)\right) \\
& =\sum_{i \in N}\left[p\left(\sum_{S \in \mathcal{B}_{i}} \lambda_{S} X^{P, S}\right) y_{i}-C_{i}\left(y_{i}\right)\right] \\
& \leq p(Y) Y-\sum_{i \in N} C_{i}\left(y_{i}\right) \\
& \leq v_{\gamma}(N),
\end{aligned}
$$

which completes the proof.

The concave condition in theorem 4.2 is a sufficient condition for $\gamma$-core existence, but it is not a necessary condition. This is illustrated in the following example.

\section{Example 4.4}

Consider the oligopoly TU-game $v_{\gamma} \in G_{o}^{N}$ associated with the oligopoly situation $\left(N,\left(w_{i}, C_{i}\right)_{i \in N}, p\right)$ where $N=\{1,2,3\}, w_{1}=5, w_{2}=1, w_{3}=2, C_{1}\left(x_{1}\right)=x_{1}, C_{2}\left(x_{2}\right)=2 x_{2}$, $C_{3}\left(x_{3}\right)=2 x_{3}$ and the inverse demand function is defined as $p(X)=10-X$. Clearly, every individual profit function is not concave on $X_{N}$. The worth of every coalition $S \in \mathcal{P}(N)$ is given in the following table:

\begin{tabular}{|c|ccccccc|}
\hline$S$ & $\{1\}$ & $\{2\}$ & $\{3\}$ & $\{1,2\}$ & $\{1,3\}$ & $\{2,3\}$ & $\{1,2,3\}$ \\
\hline$v_{\gamma}(S)$ & 9 & 2 & 4 & 12.25 & 16 & 5.44 & 20.25 \\
\hline
\end{tabular}

We can easily check that $\sigma=(13.25,3,4)$ is in the $\gamma$-core, and so the $\gamma$-core is nonempty.

\section{A solution in the $\gamma$-core for oligopoly TU-games with linear cost functions}

Although the concave condition seems to be a natural requirement to guarantee the non-emptiness of the $\gamma$-core, many oligopoly situations fail to satisfy it. For instance, in a linear oligopoly situation, i.e. $p(X)=a-X$ where $a \in \mathbb{R}_{++}$, and for all $i \in N$ $C_{i}\left(x_{i}\right)=c_{i} x_{i}$ where $c_{i} \in \mathbb{R}_{+}$, every individual profit function is quadratic but it is not concave on $X_{N}$ as in example 4.4. In this section, we adopt an alternative approach that consists in providing a solution in the $\gamma$-core without the concavity requirement. We 
succeed in doing that by assuming that cost functions are linear and that firms have the same marginal cost, i.e.

$$
\exists c \in \mathbb{R}_{+} \text {s.t. } \forall i \in N, C_{i}\left(x_{i}\right)=c x_{i}
$$

We do not impose any other conditions on the capacity constraints and the inverse demand function. For a fixed set of players $N$, we denote by $G_{o}^{N *}$ the set of oligopoly TUgames associated with an oligopoly situation $\left(N,\left(w_{i}, C_{i}\right)_{i \in N}, p\right)$ satisfying (30). A solution on $G_{o}^{N *}$ is a mapping $\rho: G_{o}^{N *} \longrightarrow \mathbb{R}^{n}$ that associates to every oligopoly TU-game $v_{\gamma} \in G_{O}^{N *}$ a payoff vector $\rho\left(v_{\gamma}\right) \in \mathbb{R}^{n}$. For notational convenience, given the normal form oligopoly game $\left(N,\left(X_{i}, \pi_{i}\right)_{i \in N}\right)$ we denote by $X^{P, \emptyset}=\sum_{i \in N} \tilde{z}_{i}\left(\tilde{z}_{-i}\right)$ the total output on the Nash equilibrium. For any oligopoly TU-game $v_{\gamma} \in G_{o}^{N *}$ consider the solution, called the NP(Nash Pro rata)-value, defined as follows

$$
\forall i \in N, \operatorname{NP}_{i}\left(v_{\gamma}\right)= \begin{cases}\frac{\tilde{z}_{i}\left(\tilde{z}_{-i}\right)}{X^{P, \emptyset}} v_{\gamma}(N) & \text { if } X^{P, \emptyset}>0 \\ 0 & \text { otherwise }\end{cases}
$$

The NP-value distributes to every player $i \in N$ the worth of the grand coalition, $v_{\gamma}(N)$, in proportion to his Nash individual output $\tilde{z}_{i}\left(\tilde{z}_{-i}\right)$.

Theorem 5.1 For every oligopoly TU-game $v_{\gamma} \in G_{o}^{N *}$, it holds that $N P\left(v_{\gamma}\right) \in C\left(v_{\gamma}\right)$.

Proof: First, pick any $v_{\gamma} \in G_{o}^{N *}$ such that $X^{P, \emptyset}=0$. By (i) of corollary 3.8, for every $S \in \mathcal{P}(N)$ we have $X^{P, S}=0$, and so $v_{\gamma}(S)=0$. In this case, it is obvious that $\operatorname{NP}\left(v_{\gamma}\right) \in C\left(v_{\gamma}\right)$.

Then, pick any $v_{\gamma} \in G_{o}^{N *}$ such that $X^{P, \emptyset}>0$. For the sake of contradiction assume that $\operatorname{NP}\left(v_{\gamma}\right) \notin C\left(v_{\gamma}\right)$, i.e. there exists a deviating coalition $S \in \mathcal{P}(N)$ such that $v_{\gamma}(S)>\sum_{i \in S} \mathrm{NP}_{i}\left(v_{\gamma}\right)$. It follows that $v_{\gamma}(S)>0$ and so $X^{P, S}>0$. Consider the payoff vector $\sigma_{S} \in \mathbb{R}^{n}$ defined as

$$
\forall i \in N, \sigma_{S, i}=\frac{v_{\gamma}(N)}{X^{P, S}} \alpha_{i}
$$

where

$$
\alpha_{i}= \begin{cases}x_{S, i}^{*}\left(\tilde{z}_{-S}\right) & \text { if } i \in S \\ \tilde{z}_{i}\left(x_{S}^{*}, \tilde{z}_{-S \cup i}\right) & \text { if } i \notin S\end{cases}
$$

From (i) and (ii) of corollary 3.8, we know that $X^{P, S} \leq X^{P, \emptyset}$ and $\tilde{z}_{i}\left(\tilde{z}_{-i}\right) \leq \tilde{z}_{i}\left(x_{S}^{*}, \tilde{z}_{-S \cup i}\right)$ for all $i \notin S$ respectively. This implies that $\sigma_{S, i}\left(v_{\gamma}\right) \geq \mathrm{NP}_{i}\left(v_{\gamma}\right)$ for all $i \notin S$. Moreover, by the Pareto efficiency of the worth of the grand coalition and the contradicting assumption it holds that 


$$
\begin{aligned}
\sum_{i \in S} \sigma_{S, i}\left(v_{\gamma}\right) & =\frac{v_{\gamma}(N)}{X^{P, S}} \sum_{i \in S} x_{S, i}^{*}\left(\tilde{z}_{-S}\right) \\
& \geq \frac{1}{X^{P, S}}\left(p\left(X^{P, S}\right) X^{P, S}-c X^{P, S}\right) \sum_{i \in S} x_{S, i}^{*}\left(\tilde{z}_{-S}\right) \\
& =p\left(X^{P, S}\right) \sum_{i \in S} x_{S, i}^{*}\left(\tilde{z}_{-S}\right)-c \sum_{i \in S} x_{S, i}^{*}\left(\tilde{z}_{-S}\right) \\
& =v_{\gamma}(S) \\
& >\sum_{i \in S} \operatorname{NP}_{i}\left(v_{\gamma}\right) .
\end{aligned}
$$

Thus, we obtain $\sum_{i \in N} \sigma_{S, i}\left(v_{\gamma}\right)>\sum_{i \in N} \mathrm{NP}_{i}\left(v_{\gamma}\right)$ and $\sum_{i \in N} \rho_{S, i}\left(v_{\gamma}\right)=v_{\gamma}(N)=$ $\sum_{i \in N} \mathrm{NP}_{i}\left(v_{\gamma}\right)$, a contradiction.

Note that for large capacity constraints the NP-value is equal to the Equal Division solution that distributes $v_{\gamma}(N)$ equally among the players since in this case $\tilde{z}_{i}\left(\tilde{z}_{-i}\right)=$ $\tilde{z}_{j}\left(\tilde{z}_{-j}\right)$ for all $i, j \in N, i \neq j$. Funaki and Yamato (1999) show that this latter value belongs to the $\gamma$-core of their common pool game without capacity constraints. Since their common pool game belongs to the class $G_{o}^{N *}$, theorem 5.1 generalizes their result from no capacity constraint to asymmetric capacity constraints. Thus, on the class $G_{o}^{N *}$, contrary to the Equal Division solution, the NP-value is a solution that always belongs to the $\gamma$-core by taking into account players' asymmetric capacity constraints.

From the regulator point of view it is interesting to know which properties are satisfied by the NP-value. On the class $G_{o}^{N *}$, the NP-value can be characterized by means of four properties: efficiency, null firm, monotonicity and non-cooperative fairness. For every oligopoly TU-game $v_{\gamma} \in G_{O}^{N *}$, we say that a solution $\rho$ satisfies:

1. Efficiency: if $\sum_{i \in N} \rho_{i}\left(v_{\gamma}\right)=v_{\gamma}(N)$

2. Null firm: if for all $i \in N$ such that $w_{i}=0$, then $\rho_{i}\left(v_{\gamma}\right)=0$

3. Monotonicity: if for all $i, j \in N$ such that $w_{i} \geq w_{j}$, then $\rho_{i}\left(v_{\gamma}\right) \geq \rho_{j}\left(v_{\gamma}\right)$

4. Non-cooperative fairness: if for all $i, j \in N, v_{\gamma}(\{j\}) \rho_{i}\left(v_{\gamma}\right)=v_{\gamma}(\{i\}) \rho_{j}\left(v_{\gamma}\right)$

Theorem 5.2 A solution $\rho$ on $G_{o}^{N *}$ satisfies (EFF), (NF), (M) and (NCF) if and only if $\rho=N P$.

Proof: Pick any $v_{\gamma} \in G_{o}^{N *}$. Firstly, we show that the NP-value satisfies (EFF). Assume that $X^{P, \emptyset}=0$. It follows that $\mathrm{NP}_{i}\left(v_{\gamma}\right)=0$ for all $i \in N$. Moreover, by (i) of corollary 3.8 we have $X^{P, N}=0$, and so $\sum_{i \in N} \mathrm{NP}_{i}\left(v_{\gamma}\right)=v_{\gamma}(N)=0$. Then, assume that $X^{P, \emptyset}>0$. By (31) we solve directly that $\sum_{i \in N} \mathrm{NP}_{i}\left(v_{\gamma}\right)=v_{\gamma}(N)$.

Secondly, we show that the NP-value satisfies (NF). Pick any $i \in N$ such that $w_{i}=0$. 
Assume that $X^{P, \emptyset}=0$. By $(31)$ we have $\mathrm{NP}_{i}\left(v_{\gamma}\right)=0$. Then, assume that $X^{P, \emptyset}>0$. Since $w_{i}=0$ it follows that $\tilde{z}_{i}\left(\tilde{z}_{-i}\right)=0$, and so by $(31)$ we have $\mathrm{NP}_{i}\left(v_{\gamma}\right)=0$.

Thirdly, we show that the NP-value satisfies (M). Pick any $i, j \in N$ such that $w_{j} \geq w_{i}$. Assume that $X^{P, \emptyset}=0$. By (31) we have $\mathrm{NP}_{j}\left(v_{\gamma}\right)=\mathrm{NP}_{i}\left(v_{\gamma}\right)=0$. Then, assume that $X^{P, \emptyset}>0$. From $w_{j} \geq w_{i}$ and $v_{\gamma} \in G_{o}^{N *}$ it follows that $\tilde{z}_{j}\left(\tilde{z}_{-j}\right) \geq \tilde{z}_{i}\left(\tilde{z}_{-i}\right)$, and so by (31) we have $\mathrm{NP}_{j}\left(v_{\gamma}\right) \geq \mathrm{NP}_{i}\left(v_{\gamma}\right)$.

Fourthly, we show that the NP-value satisfies (NCF). Assume that $X^{P, \emptyset}=0$. It follows that $\operatorname{NP}_{i}\left(v_{\gamma}\right)=0$ for all $i \in N$, and so $v_{\gamma}(\{j\}) \mathrm{NP}_{i}\left(v_{\gamma}\right)=v_{\gamma}(\{i\}) \mathrm{NP}_{j}\left(v_{\gamma}\right)=0$ for all $i, j \in N$. Then, assume that $X^{P, \emptyset}>0$. For all $i, j \in N$ it holds that

$$
\begin{aligned}
v_{\gamma}(\{j\}) \mathrm{NP}_{i}\left(v_{\gamma}\right) & =\left(p\left(X^{P, \emptyset}\right)-c\right) \tilde{z}_{j}\left(\tilde{z}_{-j}\right) \frac{\tilde{z}_{i}\left(\tilde{z}_{-i}\right)}{X^{P, \emptyset}} v_{\gamma}(N) \\
& =\left(p\left(X^{P, \emptyset}\right)-c\right) \tilde{z}_{i}\left(\tilde{z}_{-i}\right) \frac{\tilde{z}_{j}\left(\tilde{z}_{-j}\right)}{X^{P, \emptyset}} v_{\gamma}(N) \\
& =v_{\gamma}(\{i\}) \operatorname{NP}_{j}\left(v_{\gamma}\right) .
\end{aligned}
$$

It remains to show that the NP-value is the unique solution on the class $G_{o}^{N *}$ that satisfies (EFF), (NF), (M) and (NCF). Pick any solution $\rho$ on $G_{o}^{N *}$ satisfying (EFF), (NF), (M) and (NCF) and prove that it is equal to the NP-value. By (EFF), we know that $\sum_{i \in N} \rho_{i}\left(v_{\gamma}\right)=v_{\gamma}(N)$. Moreover, (NF) and $(\mathrm{M})$ ensures that $\rho_{i}\left(v_{\gamma}\right) \geq 0$ for all $i \in N$. Thus, there exists $\beta: G_{o}^{N *} \longrightarrow \mathbb{R}_{+}^{n}$ such that

$$
\forall i \in N, \rho_{i}\left(v_{\gamma}\right)=\frac{\beta_{i}\left(v_{\gamma}\right)}{\sum_{j \in N} \beta_{j}\left(v_{\gamma}\right)} v_{\gamma}(N)
$$

Assume that $X^{P, \emptyset}=0$. By (i) of corollary 3.8 we have $X^{P, N}=0$, and so $v_{\gamma}(N)=0$. It follows that $\rho_{i}\left(v_{\gamma}\right)=\mathrm{NP}_{i}\left(v_{\gamma}\right)=0$ for all $i \in N$. Then, assume that $X^{P, \emptyset}>0$. Without loss of generality, assume that $\sum_{i \in N} \beta_{i}\left(v_{\gamma}\right)=X^{P, \emptyset}$. By (32) and (NCF) it follows that

$$
\forall i, j \in N, v_{\gamma}(\{j\}) \beta_{i}\left(v_{\gamma}\right)=v_{\gamma}(\{i\}) \beta_{j}\left(v_{\gamma}\right) .
$$

For every $i \in N$, by summing the equations above over all $j \in N$ we obtain

$$
\begin{aligned}
\sum_{j \in N} v_{\gamma}(\{j\}) \beta_{i}\left(v_{\gamma}\right)=v_{\gamma}(\{i\}) \sum_{j \in N} \beta_{j}\left(v_{\gamma}\right) & \Longleftrightarrow\left(p\left(X^{P, \emptyset}\right)-c\right) X^{P, \emptyset} \beta_{i}\left(v_{\gamma}\right)=\left(p\left(X^{P, \emptyset}\right)-c\right) \tilde{z}_{i}\left(\tilde{z}_{-i}\right) X^{P, \emptyset} \\
& \Longleftrightarrow \beta_{i}\left(v_{\gamma}\right)=\tilde{z}_{i}\left(\tilde{z}_{-i}\right) .
\end{aligned}
$$

Thus, we conclude that $\rho_{i}\left(v_{\gamma}\right)=\operatorname{NP}_{i}\left(v_{\gamma}\right)$ for all $i \in N$. 
One can ask whether oligopoly TU-games in $\gamma$-characteristic function form are superadditive $^{8}$ or convex. ${ }^{9}$ Norde et al. (2002) establish that oligopoly TU-games in $\beta$ characteristic function form are convex in case the inverse demand function and cost functions are linear. As the following example shows, this result can not be extended for oligopoly TU-games in $\gamma$-characteristic function form.

\section{Example 5.3}

Consider the oligopoly TU-game $v_{\gamma} \in G_{o}^{N *}$ associated with the oligopoly situation $\left(N,\left(w_{i}, C_{i}\right)_{i \in N}, p\right)$ where $N=\{1,2,3\}, w_{1}=3 / 2, w_{2}=3, w_{3}=5 / 2, C_{i}\left(x_{i}\right)=2 x_{i}$ for all $i \in N$, and the inverse demand function is defined as $p(X)=10-X$. The worth of every coalition $S \in \mathcal{P}(N)$ is given in the following table:

\begin{tabular}{|c|ccccccc|}
\hline$S$ & $\{1\}$ & $\{2\}$ & $\{3\}$ & $\{1,2\}$ & $\{1,3\}$ & $\{2,3\}$ & $\{1,2,3\}$ \\
\hline$v_{\gamma}(S)$ & 3.25 & 4.69 & 4.69 & 7.56 & 7.56 & 10.56 & 16 \\
\hline
\end{tabular}

Thus, $v(\{1,2\})<v(\{1\})+v(\{2\})$. We conclude that the oligopoly TU-game $v_{\gamma} \in G_{o}^{N *}$ is neither superadditive nor convex.

\section{Concluding remarks}

In this paper, we have focused on oligopoly TU-games in $\gamma$-characteristic function form. When a coalition forms, the underlying assumption is that external agents choose their action individually as a best reply to the coalitional action (Chander and Tulkens 1997; Helm 2001). For the class of oligopoly TU-games, this assumption seems more appropriate than the standard assumption for which those external agents act to minimize the coalitional profit and used to define the $\beta$-characteristic function. Oligopoly TU-games in $\beta$-characteristic function form were studied, among others, by Zhao (1999a,b), Norde et al. (2002), Driessen and Meinhardt (2005). Unsurprisingly, the $\gamma$-core is included in the $\beta$-core.

In order to verify that the $\gamma$-characteristic function is well-defined, we have proved that an equilibrium under every coalition structure, and so every partial agreement equilibrium, exists. We have studied the variations of equilibrium outputs of every coalition according to the coarseness of the coalition structure in which it is embedded. We have showed that total production equilibrium is decreasing with the coarseness of the coalition structure. This result is explained by the mergers between coalitions that occurred. Conversely, the other coalitions which do not merge increase their output.

Concerning the non-emptiness of the $\gamma$-core, we have first established that oligopoly TUgames in $\gamma$-characteristic function form are balanced when the inverse demand function

\footnotetext{
${ }^{8} \mathrm{~A}$ TU-game $v \in G^{N}$ is superadditive if for every $S, T \in \mathcal{P}(N)$ such that $S \cap T=\emptyset$ it holds that $v(S)+v(T) \leq v(S \cup T)$.

${ }^{9} \mathrm{~A}$ TU-game $v \in G^{N}$ is convex if for every $S, T \in \mathcal{P}(N)$ it holds that $v(S)+v(T) \leq v(S \cup T)+$ $v(S \cap T)$.
} 
is differentiable and every individual profit function is continuous and concave on the set of strategy profiles. As already discussed earlier, this result is a step forward beyond Zhao's theorem (1999b) for the class of oligopoly TU-games. However, many oligopoly situations fail to satisfy the concavity condition. In particular, this is the case when firms have linear cost functions. Thus, in case of the cost functions are linear and firms have the same marginal cost, we have introduced a single-valued allocation rule, the NP-value, that distributes to every player the worth of the grand coalition in proportion to his Nash individual output. We have showed that this solution belongs to the $\gamma$-core. Insofar as our oligopoly game also describes a common pool game, this result generalizes Funaki and Yamato's core existence result (1999) from no capacity constraint to asymmetric capacity constraints. Moreover, we have provided an axiomatic characterization of the NP-value by means of four natural properties.

van den Brink (2008) proposes an axiomatic characterization of a class of proportional solutions with exogenous weights. Define $\Delta^{n}=\left\{\lambda \in \mathbb{R}_{+}^{n}: \sum_{i \in N} \lambda_{i}=1\right\}$ the $(n-1)$ dimensional unit simplex. For every $\lambda \in \Delta^{n}$ and every TU-game $v \in G^{N}$ a proportional solution, $\rho^{\lambda}(v)$, is defined as $\rho_{i}^{\lambda}(v)=\left(\lambda_{i} / \sum_{j \in N} \lambda_{j}\right) v(N)$ for all $i \in N$. van den Brink (2008) characterizes this class of proportional solutions with three axioms: efficiency, collusion neutrality ${ }^{10}$ and linearity. We know that the NP-value is an efficient solution on $G_{o}^{N *}$. However, it fails to satisfy collusion neutrality and linearity. We have characterized a proportional solution with endogenous weights (Nash individual outputs) for the particular class $G_{o}^{N *}$. An axiomatic characterization of a proportional solution with endogenous weights on the general class $G^{N}$ would be of the greatest interest.

Other alternative blocking rules can be considered. For instance, agents in $N \backslash S$ can choose coalitional (rather than individual) best reply strategies. In this case, in order to determine the worth of every coalition $S \in \mathcal{P}(N)$, the equilibrium under the coalition structure $\mathcal{P}=\{S, N \backslash S\}$ must be considered. However, Funaki and Yamato (1999) show that the associated core of their common pool game is always empty for $n \geq 4$ in case of the cost functions are symmetric and linear. This result remains valid for our model.

\section{References}

Aumann, R. (1959). Acceptable Points in General Cooperative n-Person Games, in: Tucker, luce (eds.), contributions to the theory of games iv. Annals of Mathematics Studies Vol. 40, Princeton University Press, Princeton.

Bondareva, O. N. (1963). Some applications of linear programming methods to the theory of cooperative games. Problemi Kibernetiki 10, 119-139.

\footnotetext{
${ }^{10} \mathrm{~A}$ solution $\rho: G^{N} \longrightarrow \mathbb{R}^{n}$ satisfies collusion neutrality if $\rho_{i}\left(v_{i j}\right)+\rho_{j}\left(v_{i j}\right)=\rho_{i}(v)+\rho_{j}(v)$ for every $i, j \in N$ and every $v \in G^{N}$ where for every $S \in \mathcal{P}(N)$, we have

$$
v_{i j}(S)= \begin{cases}v(S \backslash\{j\}) & \text { if } i \notin S, \\ v(S \cup\{j\}) & \text { if } i \in S .\end{cases}
$$
}


Chander, P. and H. Tulkens (1997). A core of an economy with multilateral environmental externalities. International Journal of Game Theory 26, 379-401.

Driessen, T. S. and H. I. Meinhardt (2005). Convexity of oligopoly games without transferable technologies. Mathematical Social Sciences 50, 102-126.

Funaki, Y. and T. Yamato (1999). The core of an economy with a common pool resource: A partition function form approach. International Journal of Game Theory $28,157-171$.

Helm, C. (2001). On the existence of a cooperative solution for a coalitional game with externalities. International Journal of Game Theory 30, 141-146.

Kaneko, M. (1977). The ratio equilibria and the core of the voting game $g(n, w)$ in a public goods economy. Econometrica 45, 1589-1594.

Moulin, H. and A. Watts (1997). Two versions of the tragedy of the commons. Economic Design 2, 399-421.

Norde, H., K. H. P. Do, and S. Tijs (2002). Oligopoly games with and without transferable technologies. Mathematical Social Sciences 43, 187-207.

Okuguchi, K. and F. Szidarovszky (1990). The theory of oligopoly with muti-product firms. springer-Verlag, Berlin.

Rosenthal, R. (1971). External economies and cores. Journal of Economic Theory 3, 182-188.

Shapley, L. S. (1967). On balanced sets and cores. Naval Research Logistics Quaterly 14, 453-460.

van den Brink, R. (2008). Efficiency and collusion neutrality of solutions for cooperative tu-games.

Zhao, J. (1999a). A necessary and sufficient condition for the convexity in oligopoly games. Mathematical Social Sciences 37, 189-204.

Zhao, J. (1999b). A $\beta$-core existence result and its application to oligopoly markets. Games and Economic Behavior 27, 153-168. 